\title{
Diseño y evaluación de consolidantes para el patrimonio pétreo de origen volcánico
}

\author{
Nora Ariadna Pérez Castellanos \\ Enrique Lima Muñoz
}

I patrimonio pétreo es uno de los más abundantes en México, presente en la arquitectura prehispánica, colonial y moderna, así como en multitud de bienes muebles adosados, asociados o exentos.

Actualmente, la conservación-restauración del patrimonio es un campo profesional de las ciencias sociales que tiene un nexo muy estrecho con las ciencias exactas debido, entre otras razones, a que para lograr la preservación de la materialidad del bien cultural es necesario comprender y estudiar la transformación de los materiales a través del tiempo. Asimismo, las ciencias básicas colaboran en el desarrollo y evaluación de nuevas tecnologías en materia de intervención en objetos de valor cultural.

Uno de los principales procedimientos que los conservadores-restauradores Ilevan a cabo en los bienes culturales pétreos disgregados es la consolidación, que se realiza básicamente para restablecer la estabilidad estructural y mejorar la resistencia mecánica del sustrato que ha sido afectado por distintos agentes de alteración, como la contaminación atmosférica, la cristalización de sales, la humedad, los factores biológicos y las acciones antropogénicas.

A lo largo del tiempo, los conservadores-restauradores han empleado distintos tipos de consolidantes: inorgánicos (p. e., hidróxido de bario, silicatos), orgánicos naturales (v. gr., el mucílago o la goma de nopal) (Jáidar 2006:105) o sintéticos (resinas epóxicas y acrílicas) (Selwitz 1992:7). Los consolidantes más empleados en la actualidad se basan en los alcoxisilanos, principalmente en el tetraetoxisilano (por su símbolo, TEOS) y sus olígomeros (Price y Doehne 2006:17; Wheeler 2005:13).

Evaluar el desempeño de los distintos consolidantes, en particular de los recientemente desarrollados, es muy importante para seleccionar adecuadamente el que ha de emplearse. Ello se debe en gran medida a la irreversibilidad que implica el proceso de consolidación, de manera que contar con los registros científicos del comportamiento del sustrato ya consolidado, tanto a corto como largo plazo, es indispensable para asegurar que la intervención no se convertirá en una nueva causa de alteración. 
Se sabe que, en razón de que en México todavía no se ha desarrollado un campo profesional para el diseño y la síntesis de materiales para tratamientos de conservación-restauración de bienes culturales pétreos, muchos de los productos usados en la intervención se producen en el extranjero (Estados Unidos y Europa) y, por lo tanto, tienen que importarse, las más de las veces a un alto costo. Adicionalmente, muchos de los consolidantes importados no son pertinentes para nuestro país, ya que su desempeño corresponde a condiciones climáticas distintas de aquellas a las que se ve expuesto el patrimonio pétreo mexicano, y en muchos casos no es posible modificarlos para adaptarlos a las necesidades nacionales, ya que no se conoce su composición exacta.

En el campo de la conservación en México se ha estudiado la aplicación de varios materiales sobre rocas de origen sedimentario, como por ejemplo el hidróxido de bario (Anrubio 1996:68) y el fluoruro de sodio (Straulino 2010:41), ambos en piedra caliza, o los alcoxisilanos en estucos (Mendiolea 1985:37), mientras que para las rocas de origen volcánico se ha reportado el estudio del sílice coloidal (Lugo 2007:27) y la evaluación de Paraloid $\mathrm{B} 72^{\odot}$ y $\mathrm{KSE} 300^{\circ}$ como fijador de pigmentos en roca andesítica (Barragán y Malváez 2010:52). A escala internacional, para las tobas volcánicas aún se informa del uso de los alcoxisilanos (Ziegenbalg y Piaszczynski 2012; Di Benedetto et al. 2012).

En el occidente de México existe gran diversidad de inmuebles construidos con toba volcánica, por lo que se consideró pertinente desarrollar un consolidante que fomentara la estabilidad material de este tipo de piedra en condiciones climáticas de la región. Los consolidantes sintetizados se compararon con el comercial: $\operatorname{KSE~} 300^{\circ}{ }^{1}$ que se aplicaron en probetas alteradas recuperadas de un edificio histórico, para lo cual se tomó como caso de estudio la toba volcánica del Templo de Santa Mónica en Guadalajara, Jalisco. Las propiedades de las probetas consolidadas se evaluaron y compararon con las de piedra nueva, extraídas directamente de la cantera.

El desarrollo, resultados y discusión de este estudio, realizado por profesionales de las ciencias de materiales, es el objeto del presente artículo.

\section{Metodología}

La metodología de investigación constó de tres etapas. La primera fue la caracterización de la piedra, que tuvo como finalidad tanto conocer el material que había de consolidarse como establecer su grado de deterioro; la

\footnotetext{
${ }^{1}$ Se decidió hacer una evaluación comparativa del producto comercial KSE $300^{\circ}$ de Remmers debido a que fue el consolidante que se empleó en la restauración del Templo de Santa Mónica, además de que se usó en otros proyectos para toba volcánica. Su composición reportada es: consolidante de piedra exento de disolventes sobre la base de etil éster del ácido silícico (Remmers 2013).
}

segunda consistió en la síntesis y caracterización del consolidante de tipo alcoxisilano y, finalmente, esta sustancia se aplicó en piedras alteradas y se evaluaron sus propiedades.

Para este estudio se tomaron muestras de $15 \mathrm{X} 15 \mathrm{X}$ $15 \mathrm{~cm}$ de piedras deterioradas de color amarillo y blan$\mathrm{co}$, a partir de aquellas que se retiraron de acuerdo con el criterio establecido por el proyecto de restauración del Templo de Santa Mónica, edificación de estilo barroco salomónico construida entre 1690 y 1720, como parte del convento de las monjas agustinas recoletas de clausura (Jáuregui 2008:61). Durante el proceso de restauración se removieron piedras, previamente desalinizadas, que afectaban la estabilidad del inmueble y tenían diferentes tipos de alteración. Las piedras removidas se reemplazaron por otras (frescas) de la cantera original, es decir, con aquellas con las que se construyó el templo (Jáuregui 2010: comunicación personal); en seguida, se tomaron muestras de estas últimas, nuevamente de $15 \times 15 \times 15 \mathrm{~cm}$ de cada color.

Las probetas se clasificaron como piedra deteriorada blanca (PDB) y piedra deteriorada amarilla (PDA), para las obtenidas del templo, y como piedra nueva blanca (PNB) y piedra nueva amarilla (PNA), para las recién extraídas de la cantera.

Se hizo un estudio completo para identificar los deterioros de las PDB y PDA, mediante el cual se determinó que éstas sufrieron un proceso de erosión e intemperismo que causó, por una parte, la disgregación del dióxido de silicio amorfo en la matriz y, por la otra, que el feldespato (sanidina) quedara en superficie, lo que propició, en el caso de la piedra amarilla, un proceso de hidrólisis del silicato y la extracción preferencial de $\mathrm{SiO}_{2}$ (Pérez CasteIlanos y Lima Muñoz 2012).

Con base en estos resultados, se sintetizaron, por el método de sol-gel: silicato y aluminosilicato, dos variaciones de consolidantes, las cuales se compararon con el producto comercial alemán KSE $300^{\circ}$ de la marca Remmers $^{\odot}$. Los consolidantes se caracterizaron por los métodos de adsorción de nitrógeno y diversas espectroscopias; por mencionar algunas: resonancia magnética nuclear con giro al ángulo mágico (RMN-MAS) de los núcleos ${ }^{29} \mathrm{Si}$ y ${ }^{27} \mathrm{Al}$, y espectroscopia infrarroja por transformada de Fourier con el método de reflectancia atenuada (FTIR-ATR). Se les realizaron análisis termogravimétricos (TGA) y microscopia electrónica de barrido (MEB); además, se midieron su viscosidad dinámica y el ángulo de contacto.

Por medio de papetas, ${ }^{2}$ se consolidaron grupos de tres probetas con dimensiones de $5 \times 2 \times 2 \mathrm{~cm}$ con el silicato y consolidante aluminosilicato sintetizado, así

\footnotetext{
${ }^{2}$ Se humectó pulpa de celulosa con cada consolidante y posteriormente se aplicó en una de las caras superiores de la probeta, cada una de las cuales se mantuvo dentro de bolsas de plástico para prevenir la rápida evaporación de los consolidantes. Se seleccionó este método en razón de que es de uso frecuente en procesos de restauración.
} 
como con $\mathrm{KSE} 300^{\circ}$. Con las técnicas anteriormente señaladas se evaluó el desempeño de los consolidantes en las muestras, aunado al análisis de sus propiedades macroscópicas, tales como la resistencia a la compresión (de acuerdo con la norma ASTM D2938-95) y el color (mediante el uso de un espectrómetro).

Con la finalidad de hacer una evaluación a mediano plazo del desempeño del consolidante, las piedras consolidadas se sometieron a un proceso de envejecimiento acelerado durante tres meses - el equivalente a tres años, aproximadamente, de exposición en condiciones ambientales estándares - en una cámara con condiciones especiales de luz UV, temperatura y humedad (para más información respecto de las técnicas empleadas, véase Pérez Castellanos 2012).

En las siguientes secciones se presentarán, usando la nomenclatura correspondiente, los resultados más relevantes de la caracterización de consolidantes y de la evaluación del desempeño en muestras.

\section{Caracterización de los consolidantes}

Los consolidantes de tipo silicato y aluminosilicato que se sintetizaron están diseñados para reaccionar, por medio de un catalizador, con las moléculas de agua o grupos hidroxilo $\left(\mathrm{OH}^{-}\right)$que se encuentran en la piedra, lo que promueve la polimerización ${ }^{3}$ del consolidante para formar una estructura tridimensional no cristalina de silicato o aluminosilicato, que sirve para optimizar la estabilidad estructural y la resistencia mecánica del sustrato.

Los consolidantes que se encuentran en fase líquida, para pasar a la fase sólida tienen una etapa intermedia que es la formación del xerogel ${ }^{4}$ durante la cual se produce como producto secundario un alcohol que se evapora rápidamente y no permanece en la piedra. Así, este sistema es idóneo para la conservación, ya que evita la acumulación de productos secundarios que eventualmente reaccionarían más tarde o limitarían procesos de difusión.

Todos los consolidantes en solución tienen $\mathrm{pH} 5$ y son solubles en etanol, butanol y acetona, en caso de que requieran removerse previamente a la fase de gelación de alguna superficie, y, una vez secos, tienen apariencia vítrea a la escala del microscopio óptico.

Una vez formada la fase sólida, el consolidante polimérico inorgánico se considera como un equivalente amor-

\footnotetext{
${ }^{3}$ El consolidante está constituido por monómeros de alcóxidos de silicio (silicato) y alcóxidos de aluminio (aluminosilicatos), que, al reaccionar con el catalizador, moléculas de agua y grupos hidroxilo, empiezan a unirse y a formar un polímero tridimensional de $\mathrm{SiO}_{2}$. Debido a que no se aplican calor ni presión, la estructura tridimensional que se forma es no cristalina.

${ }^{4}$ Gel que se ha secado en condiciones de evaporación convencional (condiciones ambientales) (Wright 2001:29).
}

$\mathrm{fo}^{5}$ de los minerales geológicos, ${ }^{6} \mathrm{y}$, sintetizado, como polímero (Khale y Chaudhary 2007). Los compuestos sintetizados tienen características similares a los productos comerciales $\mathrm{KSE} 300^{\circ}$, Wacker $\mathrm{OH}^{\odot}$, Tegovakon $\mathrm{V}^{\odot}$ y Keim $\mathrm{OH}^{\odot}$, aunque éstos sólo forman silicatos.

En la Figura 1 se compara el espectro de FTIR de la piedra nueva blanca (PNB) con los de los sólidos que se obtuvieron de los consolidantes, donde se observa una mayor similitud con el espectro del consolidante aluminosilicato, a seguir:

- La banda principal en $997 \mathrm{~cm}^{-1}$, que corresponde a la elongación del tetraedro interno de $\mathrm{Si}-\mathrm{O}$ o Al-O, se encuentra tanto en PNB como en aluminosilicato; la diferencia más notable es en el hombro de esta banda, que para la PNB se localiza en $1124 \mathrm{~cm}^{-1}$, y en 1164 $\mathrm{cm}^{-1}$ para el aluminosilicato.

- La banda correspondiente a la apertura del poro del PNB $\left(400 \mathrm{~cm}^{-1}\right)$ también es reproducida por los consolidantes sintetizados y el KSE $300^{\circ}$. No obstante, restos de cadenas orgánicas se mantienen sólo en el KSE $300^{\circ}$ y el silicato, lo que hace más compatible el aluminosilicato con la piedras.

- En los espectros es evidente que las bandas de vibraciones características del material original en la región de $668-486 \mathrm{~cm}^{-1}$ no fueron reproducidas por los consolidantes.

Finalmente, en los espectros de IR de los consolidantes sometidos a cámara de envejecimiento acelerado ya no se presentaron las bandas correspondientes a grupos orgánicos, y disminuyó la intensidad tanto de las relativas al Si-OH y al enlace Si-O como la de elongación simétrica entre tetraedros -ésta significativamente-, lo que indica que el ambiente químico es más homogéneo.

Por su parte, en las micrografías (Figuras 2 y 3 ) se advierte que:

- Tanto los consolidantes sintetizados como el comercial tienen partículas heterogéneas. Sin embargo, el KSE $300^{\circ}$ tiene partículas de mayor tamaño que las silicato, el cual sería su similar por la composición.

- Tanto los consolidantes sintetizados como el comercial presentan fracturas.

- El consolidante tipo aluminosilicato tiene partículas de forma alargada, y se observa el arreglo de las partículas por su configuración prismática -la forma de los cristales del consolidante es importante porque determina si va a causar esfuerzos internos al penetrar en los poros-. Sin embargo, es esencial considerar que, en estas muestras, el consolidante no tiene ninguna restricción espacial, como lo tendría en su uso como consolidante de piedra.

\footnotetext{
${ }^{5}$ Estructura no cristalina.

${ }^{6}$ Los minerales, por definición, poseen una estructura cristalina.
} 


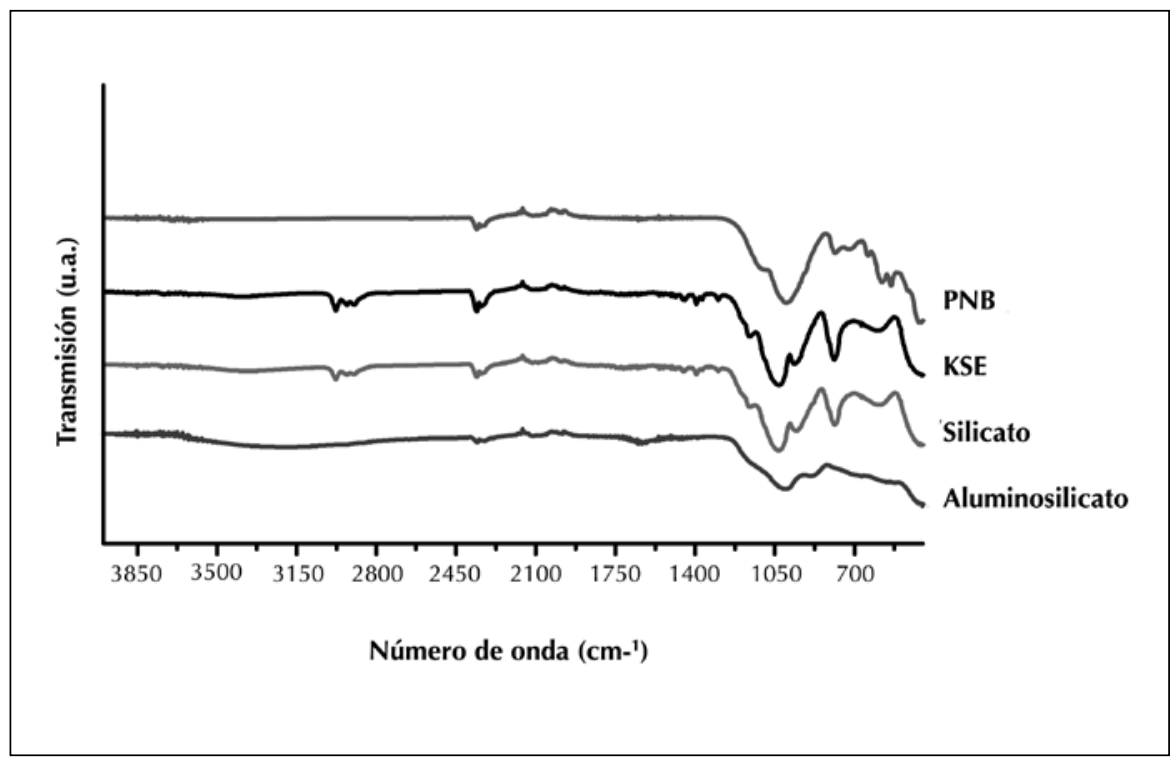

FIGURA 1. Espectro de FTIR de los consolidantes en sólido y de la piedra nueva blanca (PNB) (Cortesía: Nora Pérez, 2011).

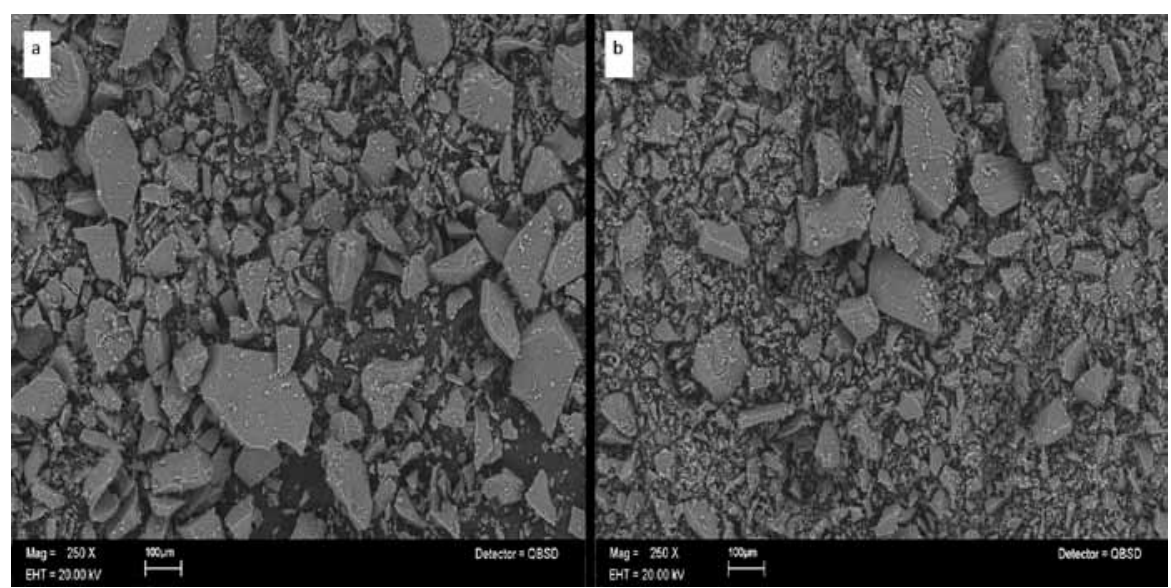

FIGURA 2. Imágenes del meb de los consolidantes: a. KSE 300 , b. silicato (Cortesía: Nora Pérez, 2011).
La técnica de adsorción de nitrógeno por el método de BET (BrunauerEmmett-Teller) reportó el mismo tipo de isotermas de adsorción tanto en los consolidantes como en la piedra natural, por lo que todos son texturalmente compatibles respecto de este mecanismo de fisisorción. ${ }^{7}$ En razón de que los consolidantes sintetizados son mesoporosos, ${ }^{8}$ con una zona específica BET mayor que la del producto comercial KSE $300^{\circ}$, se concluye que poseen una mayor superficie de reacción con el sustrato pétreo. Puesto que el radio del poro de todas las muestras consolidadas es cercano a la PNB (1.88 nm), se determina que la aplicación de estos productos a la piedra no genera alteraciones en el mecanismo de fisiosorción de ésta.

El que todos los consolidantes hayan reportado un ángulo de contacto con la piedra cercano a cero indica que esta propiedad no fue cuantificable debido a que la gota no se forma del todo, ya que la superficie del sustrato es completamente afín a los consolidantes, lo que hace posible una absorción inmediata.

Finalmente, la gráfica de esfuerzo de corte reportó una función lineal de la velocidad de corte para todos los consolidantes, lo que muestra que son fluidos newtonianos. El valor de la viscosidad para los consolidantes sintetizados fue de $1.2 \mathrm{cP}$, mientras que para el producto comercial (KSE $300^{\circ}$ ) es de 1.9 centipoises.

\section{Evaluación de los consolidantes}

La técnica de RMN-MAS fue determinante para evaluar los cambios

\footnotetext{
${ }^{7}$ Fenómeno de adsorción sin la existencia de un enlace químico; se contrapone a la quimisorción, donde éste sí existe. Las fuerzas de fisisorción son las responsables de la condensación de los vapores en los materiales y están condicionadas a la porosidad intrínseca del material (Rouquerol 1999:6).

${ }^{8}$ Materiales con poros de ancho interno entre 2 y $50 \mathrm{~nm}$ (Rouquerol 1999:8).
} 
estructurales en las probetas de las muestras de piedra consolidada en razón de que su análisis es sensible a los ambientes químicos de los átomos de Si y Al. Así, con base en la relación de las intensidades de las señales de los espectros resultantes, se infirió que:

- El consolidante aluminosilicato se distribuyó de manera homogénea en la PDB, ya que no se detectaron unidades tetraédricas de Si enriquecidas en $\mathrm{Al}$.

- Las muestras consolidadas con $\mathrm{KSE} 300^{\circ}$ y silicato presentaron, al primero y al tercer mes de envejecimiento, la misma proporción de unidades condensadas de la red de $\mathrm{SiO}_{2}$, por lo que se concluyó que consolidaron de una manera similar.

Las micrografías (Figura 4) también arrojaron resultados de importancia:

- En la PNB consolidada con KSE $300^{\circ}$ se nota que el consolidante quedó en superficie: un recubrimiento que forma mallas de $\mathrm{SiO}_{2}$, fenómeno que no se presenta en la PDA consolidada con el mismo material, en la cual, además de que la superficie es homogénea, se encuentran menos espacios vacíos.

- En ninguna de las muestras consolidadas con silicato y aluminosilicato es posible distinguir el material consolidante del sustrato: se aprecia cómo éste rellenó homogéneamente los poros de la piedra, disminuyendo su porosidad sin sellarlos, al menos a la escala micrométrica.

Cabe señalar que, respecto de las probetas de referencia, las muestras consolidadas no variaron los valores de densidad, de $1.6 \mathrm{~g} / \mathrm{cm}^{3}$ para la PNB, y de $1.8 \mathrm{~g} / \mathrm{cm}^{3}$ para la PNA.

En relación con la macroporosidad, ${ }^{9}$ se reportaron los siguientes resultados:

- En el caso de PDA: su consolidante más efectivo fue el silicato, ya que disminuyó la porosidad de la piedra, sin que ello impidiera la absorción de agua.

- En el caso de PDB: el KSE $300^{\circ}$ redujo su porosidad y prácticamente no absorbió agua; el silicato la aumentó ligeramente, pero disminuyó la velocidad de absorción de agua, mientras que el aluminosilicato mantuvo la porosidad original y su velocidad de absorción de agua fue menor.

\footnotetext{
${ }^{9}$ Relación de volumen de los espacios vacíos entre partículas y poros con acceso a la superficie del material respecto del volumen ocupado por el sólido (Rouquerol 1999:8).
}

Como resultado de las pruebas de compresión en muestras consolidadas (Figura 5), se obtuvieron los siguientes registros:

- El tratamiento con silicato generó una capacidad de carga mayor a la presentada con otros consolidantes, la cual alcanzó valores cercanos a las piedras nuevas.

- El aluminosilicato aumentó ligeramente la capacidad de carga de todas las piedras.

Dos características especiales, notables en las pruebas de compresión, fueron la homogeneidad en el patrón de ruptura y la capacidad de carga para cada serie de probetas. A pesar de que la piedra seguía siendo heterogénea, por sus cargas, las probetas tuvieron comportamientos similares en cada serie, lo cual significa que los consolidantes homogeneizan su superficie, distribuyendo los esfuerzos y haciéndola más resistente.

Finalmente, la evaluación de color de las piedras consolidadas reportó que, de acuerdo con el sistema CIE $\left[\mathrm{L}^{*} \mathrm{a}^{*} \mathrm{~b}^{*}\right]$, todas las muestras se encuentran en una misma región de color. Adicionalmente, al comparar los valores de las PDB consolidadas con los de las PDB sin consolidación, se advirtió que:

- El KSE $300^{\circ}$ opaca la superficie e intensifica las componentes verde y amarillo.

- El silicato hace a la piedra un poco más luminosa, roja y amarilla.

- El aluminosilicato produce los cambios más significativos: la piedra es más luminosa, roja y amarilla.

En la PDA se observaron mayores cambios de color como producto de la consolidación, debido a su alto contenido de óxidos de hierro, compuestos que, dependiendo del consolidante, interaccionaron de forma diferencial con la luz, a seguir:

- El KSE $300^{\circ}$ y el silicato tuvieron un mayor efecto en la piedra amarilla, haciéndola más azul y verde 


\begin{tabular}{|c|c|c|c|c|c|}
\hline $\begin{array}{c}\text { CARGA } \\
\text { MÁXIMA }\end{array}$ & Nueva & Deteriorada & $\begin{array}{c}\text { Consolidada } \\
\text { con KSE 300 }\end{array}$ & $\begin{array}{c}\text { Consolidada con } \\
\text { silicato }\end{array}$ & $\begin{array}{c}\text { Consolidada con } \\
\text { aluminosilicato }\end{array}$ \\
\hline Blanca & 9.1 & 7.5 & 9.9 & 11.2 & 9.0 \\
\hline Amarilla & 18.1 & 4.0 & 9.4 & 14.8 & 7.5 \\
\hline
\end{tabular}

FIGURA 5. Carga máxima soportada de muestras de toba en la prueba de compresión (Cortesía: Nora Pérez, 2011).

- Aunque el aluminosilicato no alteró drásticamente los componentes de color, sí afectó la luminosidad.

En todos los casos, las diferencias de color entre las piedras deterioradas sin tratamiento y las intervenidas son menores o iguales a tres unidades, por lo que, entonces, se trata de cambios imperceptibles para el ojo humano. Es importante notar que la saturación no cambió con la aplicación de ninguno de los consolidantes, lo cual descarta que se genere una apariencia de tipo "mojado".

\section{Conclusiones}

Este estudio concluye que los consolidantes sintetizados cumplen con algunos de los requerimientos establecidos internacionalmente en materia de conservación: son compatibles fisicoquímicamente con la piedra; no alteran la apariencia física del sustrato, y pueden retirarse mientras se encuentran en fase líquida. No obstante, a largo plazo, el proceso de consolidación es irreversible.

Un aspecto relevante es que los estudios hechos en muestras consolidadas después de tres años de envejecimiento acelerado no mostraron alteraciones secundarias, lo que garantiza la estabilidad del proceso a un mediano plazo.

Aunque todos los consolidantes analizados en el estudio son compatibles con el sustrato de la toba volcánica, se informó de variantes tanto en el KSE $300^{\circ}$ como en el silicato, que reaccionaron preferencialmente con la piedra amarilla, mientras el aluminosilicato fue un mejor consolidante para la piedra blanca, puesto que la piedra, a causa de la hidrólisis ocurrida durante el mecanismo de deterioro, reaccionó más con aquel material cuya composición tenía mayor parecido con el mineral que había sido extraído. Esto se determinó mediante la técnica de RMN-MAS.

Otro aspecto digno de señalarse es que, al aplicar los consolidantes, en ningún caso se modificaron las propiedades de adsorción y desorción de la toba; sí cambiaron, sin embargo, sus características como absorbentes de la piedra respecto del agua: en el del KSE $300^{\odot}$ incluso el sustrato se tornó hidrófobo. Esta peculiaridad, aunque en primera instancia pareciera favorable, no lo es ya que disminuye la difusión del agua que se encuentra dentro de la piedra hacia el exterior, lo cual eventualmente ocasionará múltiples alteraciones.

Los resultados presentados se explican por el hecho de que el consolidante se absorbe en la superficie de la piedra y entra en los poros reponiendo el material disgregado.

Las mediciones del espesor y la homogeneidad de la capa del consolidante son propiedades que no fue posible expresar cuantitativamente, pues entre los materiales sintetizados, que tienen los mismos componentes de la piedra, existe compatibilidad.

Se debe señalar que es necesario conocer las causas de deterioro del sustrato sobre el que se desee aplicar cualquier material consolidante y realizar estudios en laboratorio con el fin de asegurar los resultados deseados, con un escalamiento que va desde las pruebas en polvo hasta el objeto a tratar, puesto que cada caso es diferente y no es posible generalizar los resultados de un producto. En este estudio no se hicieron variaciones respecto del modo de aplicación de los consolidantes, pero se debe subrayar que éste tiene un papel fundamental en los resultados del tratamiento.

Las ventajas de los consolidantes sintetizados en este experimento en relación con los comerciales son, primero, que el precio de aquéllos es menor, en razón de que se evitan los costos de importación; y, segundo, se conoce su composición exacta, lo que teóricamente (dependiendo de la piedra que se desee tratar y de las condiciones de aplicación) hace posible rediseñarlos, lo que abre nuevas posibilidades para la síntesis de consolidantes específicos para tratamientos particulares de conservaciónrestauración.

\section{Agradecimientos}

Agradecemos al doctor Omar Novelo; a los ingenieros químicos Miguel Canseco y Gerardo Cedillo, todos ellos, del IIM-UNAM, y al maestro en ciencias Marco Vera, de la UAM-I, por el trabajo técnico realizado para esta investigación. En especial, a Karla Jáuregui, de Memento Conservación Restauración, que realizó el Proyecto de Restauración del Templo de Santa Mónica. Este estudio se realizó gracias al apoyo financiero del Conacyt. 


\section{Referencias}

Anrubio Vega, Elda Justina

1996 "Causas y efectos de deterioro de los materiales arqueológicos calcáreos del sur de Quintana Roo y una propuesta para su conservación", tesis de Licenciatura en Restauración de Bienes Muebles, México, ENCRyM-INAH.

Barragán, Cristina y Claudia Malváez

2010 “Evaluación del Paraloid B72 y KSE 300 como fijativos para los pigmentos presentes en el monolito de la diosa Tlaltecuhtli del Templo Mayor", tesis de Licenciatura en Restauración de Bienes Muebles, México, ENCRyM-INAH.

Benedetto, Claudia Di, Sara Bianchin, Piergiulio Cappelletti, Abner Colella, Maurizio de Gennaro, Monica Favaro, Arianna Gambirasi, Alessio Langella, Giovanni Luca y Maria Soranzo

2012 "The Neapolitan yellow tuff and the Vicenza stone: experimental investigations about effectiveness of antisweIling treatment", ponencia presentada en el $12^{\text {th }}$ International Congress on the Deterioration and Conservation of Stone, octubre de 2012, Nueva York, Columbia University.

Jáidar Benavides, Yareli

2006 "Los extractos vegetales usados como aditivos en los morteros de cal con fines de conservación", tesis de Licenciatura en Restauración de Bienes Muebles, México, ENCRyMINAH.

Jáuregui, Karla

2008 "Memoria de los proyectos de conservación y restauración hechos en la columna y escultura de san Cristóbal", mecanoescrito, Guadalajara, Memento Conservación Restauración.

Khale, Divya y Rubina Chaudhary

2007 "Mechanism of geopolymerization and factors influencing its development: a review", Journal of Materials Science 42 (3): 729-746.

Lugo Elías, César Octavio 2007 "Estudio comparativo de morteros de hidróxido de calcio y dióxido de sílice. Materiales aplicados al tratamiento de lagunas faltantes en objetos elaborados con toba volcánica de Guadalajara", tesis de Licenciatura en Restauración de Bienes Muebles, Guadalajara, ECRO.

Mendiolea Ortega, José Manuel

1985 "La sílica coloidal como consolidante de materiales pétreos in situ", tesis de Licenciatura en Restauración de Bienes Muebles, México, ENCRyM-INAH.
Pérez Castellanos, Nora Ariadna y Enrique Lima Muñoz 2012 "Chemical evolution of the volcanic tuff from the Santa Mónica Church in Guadalajara, Mexico", en José Luis Ruvalcaba Sil, Javier Reyes Trujeque, Adrián Velázquez Castro y Manuel Espinosa Pesqueira (eds.), Cultural Heritage and Archaeological Issues in Materials Science, Nueva York, Cambridge University Press, 195-203.

Pérez Castellanos, Nora Ariadna

2012 "Conservación del patrimonio pétreo: evaluación del deterioro y proceso de consolidación de la toba volcánica del Occidente de México", tesis de Maestría en Ciencia e Ingeniería de Materiales, México, IIM-UNAM.

Price, Clifford A. y Eric Doehne 2010 Stone Conservation. An Overview of Current Research, Los Angeles, Getty Conservation Institute.

Remmers

2013 Ficha técnica. Artículo número 0720. KSE 300, documento electrónico disponible en [http://www.remmers.es/fileadmin/dam/Productos/TM/E_0720_-_11.07.pdf], consultado en febrero del 2013.

Rouquerol, Françoise, Jean Rouquerol y Kenneth Sing 1999 Adsorption by Powders and Porous Solids. Principles, Methodology and Applications, Londres, Academic Press.

Selwitz, Charles

1992 Epoxy resins in Stone Consolidation, Los Ángeles, Getty Conservation Institute.

Straulino Mainou, Luisa

2010 "Fluoruro de sodio: ¿una alternativa para la conservación de piedra caliza disgregada a través de la remineralización? El caso de la cornisa del edificio 5N2 del grupo A en Río Bec, Campeche", tesis de Licenciatura en Restauración, México, ENCRYM-INAH.

Wheeler, George 2005 Alkoxysilanes and the Consolidation of Stone (Research in Conservation), Los Ángeles, Getty Conservation Institute.

Wright, John Dalton y Nico A. J. M. Sommerdijk 2001 Sol-gel Materials: Chemistry and Applications, Florida, CRC Press.

Ziegenbalg, Gerald y Ewa Piaszczynski

2012 "The combined application of calcium hydroxide nano-sols and silicic acid ester-a promising way to consolidate stone and mortar", Ponencia presentada en el $12^{\text {th }}$ International Congress on the Deterioration and Conservation of Stone, 22-26 de octubre de 2012, Nueva York, Columbia University. 


\section{Resumen}

Esta investigación es una contribución al desarrollo de la tecnología en materiales de conservación-restauración, la cual fue llevada a cabo por mexicanos especialistas del área científica. Durante su desarrollo se realizó el diseño y evaluación de materiales consolidantes específicos para tratamiento de sustrato pétreo de origen volcánico - toba- como una estrategia para conservar bienes culturales. Como caso de estudio se tomaron muestras del Templo de Santa Mónica en Guadalajara, Jalisco. Por un lado, los productos sintetizados —materiales poliméricos inorgánicos a base de silicio y aluminio - se compararon con un producto comercial: KSE $300^{\circ}$; por el otro, además de caracterizar los consolidantes, se realizó un estudio de las propiedades de la piedra consolidada, con lo que se probó que los materiales sintetizados se desempeñaron satisfactoriamente durante el proceso de consolidación de la toba.

\section{Palabras clave}

Diseño de materiales; consolidación; toba volcánica; conservación-restauración

\section{Abstract}

This research is a contribution to the development of technology in the field of conservation-restoration materials, which was carried out by Mexican specialists in the scientific area. During the development of the project, the design and evaluation of specific consolidants for treating volcanic stone substrates - tuff- was used as a strategy to preserve cultural heritage. As a case study, samples were taken from the Santa Monica Temple in Guadalajara, Jalisco. On one hand, the synthesized products -inorganic polymeric materials, based on silicon and aluminum - were compared with a commercial product: KSE $300^{\circ}$. On the other hand, in addition to the characterization of the consolidants, further studies were made to determine the properties of the consolidated stone. This proved that the synthesized materials performed satisfactorily during the consolidation process of the volcanic tuff.

\section{Key words}

Material design; consolidation; volcanic tuff; conservation-restoration

Título en inglés: Design and Evaluation of Consolidants for Volcanic

Stone Heritage 